\title{
REDES DE SENSORES SEM FIO PARA MONITORAMENTO E DETECÇÃO DE DESLIZAMENTOS DE TERRA: UMA REVISÃO SISTEMÁTICA
}

\author{
Anderson Silvério Mendrot Filho e Denise Stringhini \\ Universidade Federal de São Paulo (UNIFESP), São José dos Campos, SP, Brasil
}

\begin{abstract}
RESUMO
Deslizamentos de terra são um dos principais tipos de desastres naturais e causam danos ambientais e sociais à população em torno de áreas de risco. Uma maneira de evitar esses danos é pelo monitoramento desses eventos. Atualmente, existe uma tendência no estudo do uso de redes de sensores sem fio de baixo custo para detectar mudanças no ambiente que possam levar a desastres. O artigo apresenta uma revisão sistemática de identificação e síntese da literatura relevante na área de redes de sensores sem fio para monitoramento e detecção de deslizamentos de terra. Com a revisão sistemática, é fornecida uma visão geral e discussão sobre o estado da arte. Finalmente, são apresentadas dificuldades e desafios relacionados às implementações de campo de redes de sensores sem fio para o problema. Os resultados demonstram que o uso dessas redes para a detecção de deslizamentos de terra é viável, mas ainda é uma área que exige muito esforço em trabalhos futuros.
\end{abstract}

\section{PALAVRAS-CHAVE}

Redes de Sensores sem Fio, Desastres Naturais, Deslizamentos de Terra, Revisão Sistemática

\section{INTRODUÇÃO}

Nos últimos anos, desastres naturais têm ganhado destaque na mídia, principalmente por seus efeitos adversos sobre a sociedade e o meio ambiente. Uma das maiores causas se relaciona a deslizamentos de terra. Sua ocorrência no Brasil é significativa: no período de 2008 a 2013, atesta-se que houve por volta de 31 mil eventos, com 103 ocorrências de óbito e 303,6 mil desabrigados (IBGE, 2014). Uma alternativa para o combate ao problema é o uso de sistemas e equipamentos que façam monitoramento de mudanças no tempo e no clima, permitindo o alerta a população em caso de risco iminente de desastre. Porém, o alto custo de equipamentos de ponta inviabiliza seu uso em regiões com poucas condições financeiras ou de difícil acesso, tornando redes de sensores sem fio cada vez mais utilizadas para esses propósitos.

Nesse contexto, surgiu o projeto Cigarra, no Instituto de Ciência e Tecnologia da Universidade Federal de São Paulo, com colaboração do Centro Nacional de Monitoramento e Alertas de Desastres Naturais (Cemaden 2019), o qual tem como objetivo desenvolver e testar uma rede de sensores sem fio de baixo custo capaz de capturar parâmetros ambientais confiáveis e úteis para a detecção de deslizamentos de terra. Para tanto, o trabalho tem como base a realização de uma revisão sistemática da literatura para avaliação da pesquisa relacionada a área, além do desenvolvimento de um protótipo de rede com o uso da plataforma Arduino e da plataforma de implementação de redes de sensores sem fio Terra IoT System (Branco et al. 2015), visando o aumento da confiabilidade da rede pelo uso de redundância de dados.

O presente trabalho apresenta uma revisão sistemática realizada a fim de fundamentar o desenvolvimento de futuros protótipos para monitoração de deslizamentos de terra. Este artigo está dividido da seguinte forma: a Seção 2 exibe a revisão sistemática realizada. A Seção 3 discute os resultados obtidos a partir da revisão sistemática. A Seção 4 apresenta uma discussão sobre os principais desafios e dificuldades na implementação de redes de sensores sem fio. Por fim, a última seção traz conclusões e direções para trabalhos futuros. 


\section{REVISÃO SISTEMÁTICA}

Esta seção exibe a revisão sistemática realizada, a qual visa analisar publicações relacionadas ao uso de redes de sensores sem fio para monitoração e detecção de deslizamentos de terra, a fim de investigar seus objetivos e metodologias, além de fazer um histórico e identificar o que tem sido utilizado em projetos da área.

A primeira etapa foi a elaboração da principal questão de pesquisa, definida como: "É possível criar uma rede de sensores de baixo custo para captar parâmetros confiáveis que possam ser usados na detecção de deslizamentos de terra?". Foram definidas também as seguintes questões secundárias (QS) de pesquisa com o intuito de restringir a questão principal:

QS1: Quais sensores, módulos e itens de hardware são utilizados na implementação destas redes?

QS2: Quais as fontes de energia utilizadas para manter o funcionamento das redes?

QS3: Quais motes e microcontroladores são utilizados nos nós das redes?

QS4: Como são as arquiteturas destas redes?

QS5: Quais formas de transmissão de dados são utilizadas na implementação destas redes?

QS6: Quais os principais desafios e dificuldades em projetos que implementaram protótipo de rede real?

$\mathrm{O}$ processo de busca se deu com uma pesquisa manual em bases de dados a partir de strings de busca formuladas. Para tanto, as fontes foram selecionadas de acordo com os seguintes requisitos:

REQ1: Incluir em suas bases publicações da área de exatas;

REQ2: Possuir mecanismos de buscas que permitam o uso de expressões lógicas e que permitam pesquisa no texto completo das publicações.

Dessa forma, foram escolhidas quatro bases que foram consideradas relevantes para a pesquisa: IEEE Xplore Digital Library (IEEE), ScienceDirect, ACM Digital Library (ACM) e Google Scholar.

Em seguida foi feita a construção da string de busca. Inicialmente, utilizou-se uma mais restrita, que continha a palavra wireless. Porém, resolveu-se utilizar uma segunda string sem a palavra wireless, de forma a incluir uma quantidade maior de artigos. O uso da primeira trouxe oito artigos que não apareceram nos resultados da segunda, e estes foram incluídos por serem relevantes ao trabalho. As strings foram:

Primeira string de busca (mais restrita): "wireless sensor network" AND landslide;

Segunda string de busca (menos restrita): "sensor network" AND landslide.

No caso da IEEE e da ScienceDirect, as buscas foram realizadas pelo título, resumo e termos indexados. Por sua vez, na ACM foi necessário analisar todo o texto, pois a base não contém a opção utilizada nas duas bases anteriores. A Tabela 1 exibe a quantidade de artigos retornados pelas pesquisas nas três bases.

A revisão sistemática inicialmente foi realizada incluindo artigos até 2017, mas artigos do ano de 2018 foram acrescentados posteriormente visando a verificação de tecnologias e projetos mais recentes na área.

Tabela 1. Número de artigos por string de busca de 2005 a 2018

\begin{tabular}{cccc}
\hline & IEEE & ScienceDirect & ACM Digital Library \\
\hline Primeira string de busc: & 64 & 11 & 48 \\
Segunda string de busc: & 78 & 14 & 61 \\
Total por base & 141 & 24 & 108 \\
\hline
\end{tabular}

Foi utilizado também o Google Scholar para consolidar a busca e eventualmente trazer alguns artigos relevantes, com tal busca sendo realizada com a string de busca sensor network landslide. Houve a análise dos 100 artigos retornados pelas dez primeiras páginas, e dez deles foram incluídos para a etapa posterior por serem referentes a projetos de implementação de redes de sensores sem fio em ambientes reais.

Em seguida, foram escolhidos artigos a serem excluídos ou mantidos para a pesquisa por critérios definidos. Para o processo de inclusão, foram selecionados artigos que cumpriam com os requisitos:

REQ1: Artigos completos que foram publicados em periódicos

REQ2: Artigos escritos em inglês

REQ3: Artigos que descrevem ou especificam os sensores e/ou módulos utilizados no protótipo de nó

REQ4: Artigos que descrevem um sistema que utiliza protótipos de nó para implementação de rede visando a detecção de deslizamentos de terra

Já o processo de exclusão ocorreu em três etapas. A primeira foi a exclusão dos artigos duplicados entre as buscas e de artigos inacessíveis na instituição e de resultados que representavam descrições de anais de eventos. A segunda etapa envolveu a análise do título, resumo e caso necessário o texto como um todo. Por fim, a 
terceira etapa considerou a leitura detalhada de cada artigo. Ao final do processo de exclusão, restaram 43 artigos da IEEE, 7 da ScienceDirect, 9 da ACM Digital Library e 3 do Google Scholar.

Em seguida, foi projetada uma planilha com informações julgadas relevantes para a busca. Como nos resultados apareceram artigos relativos ao mesmo projeto, então eles foram considerados de forma conjunta, tornando a análise restrita a 48 projetos. A tabela da revisão sistemática pode ser verificada em: https://docs.google.com/spreadsheets/d/1Z1sq5buH9ta3QRqTgHTn4TAQtsOFUqiA7u47XrHZJ2k.

\subsection{Processo de Análise de Dados}

O próximo passo foi a análise de dados dos projetos a partir de diversos fatores, como transmissão de dados, sensores e microcontroladores utilizados, fornecimento de energia e topologia de rede.

Em relação a transmissão de dados, verificou-se que há projetos que utilizam mais de uma forma de transmissão. Esse uso diverso ocorre, por exemplo, se os nós sensores transmitem seus dados de uma forma entre si e de forma diferente para um gateway, ou acontece na comunicação entre gateway e centro de monitoramento. Os resultados mostraram que 33.3\% dos projetos usam rádio, o que mostra sua importância em redes de sensores sem fio no período estudado. Porém, não especificam se utilizam tecnologias ZigBee, Wi-Fi ou Bluetooth, as quais são também padrões de comunicação sem fio via rádio entre dispositivos. Outro ponto importante é a grande quantidade de comunicações via rede celular, utilizadas em 54.2\% dos projetos. Normalmente, estas tecnologias são utilizadas para a transmissão de longa distância dos dados capturados pelas redes de sensores. Já as comunicações via cabo e internet foram alternativas utilizadas em projetos em que o gateway e a central de monitoramento não estavam muito distantes.

O estudo permitiu concluir que $29 \%$ dos projetos não especificaram a forma de fornecimento de energia. Dentre os que especificaram praticamente todos utilizam bateria, com ou sem o fornecimento de energia solar. Mais especificamente, tem-se que $35.4 \%$ dos projetos utilizam somente baterias e $8.3 \%$ apenas energia solar, além de $27.1 \%$ conterem ambos. O uso de energia solar com baterias se dá, principalmente, em projetos que utilizam células ou painéis solares para carregamento de baterias recarregáveis. Outro uso ocorre no caso em que os nós sensores são abastecidos por bateria e o gateway por energia solar.

$\mathrm{Na}$ extração dos dados, verificou-se que quase todos os projetos utilizam mais de um tipo de sensor. Em relação aos parâmetros medidos para a deteç̧ão de deslizamentos, é possível perceber que os projetos levam em consideração a umidade do solo, pois 39.6\% utilizam sensores de umidade do solo, além de $20.8 \%$ utilizarem pluviômetros. Há também quatro projetos que utilizam sensores para detecção do nível da água.

Outro ponto importante é a medição da inclinação e vibração do solo, e nesse contexto os inclinômetros e acelerômetros aparecem como sensores ideais, utilizados em $35.4 \%$ e $39.6 \%$ dos projetos, respectivamente. Outro sensor bem utilizado é o extensômetro (strain gauge), aplicado em $29.1 \%$ dos projetos e responsável por medir, por exemplo, a tensão superficial de encostas. O geofone se mostrou menos utilizado, estando presente em $12.5 \%$ dos projetos; um possível motivo para isso pode ser seu custo mais elevado em relação a outros sensores. Por fim, o piezômetro, que detecta pressão de umidade do solo, também foi parte integrante de $12.5 \%$ dos projetos. Outros sensores também estiveram presentes em projetos, porém em menor quantidade, como o anemômetro e o sensor de umidade do ar.

Dentre as topologias dos projetos destaca-se a topologia em malha (mesh), utilizada em $27.1 \%$ deles, diferentemente das topologias estrela e árvore, que foram utilizadas em, respectivamente, $8.3 \%$ e $6.25 \%$ dos projetos cada uma. Além disso, $58.3 \%$ dos projetos não especificaram a rede utilizada.

Em relação a microcontroladores, $14.6 \%$ dos projetos utilizam mais de um tipo de microcontrolador ou mote. O mote MICAz, composto pelo ATmega128L e chip de rádio CC2420, é o mais utilizado, estando presente em $12.5 \%$ dos projetos. Tal mote é designado para redes de sensores sem fio de baixo consumo de energia, e possui interfaces para conexão de uma larga variedade de periféricos, além de uma plataforma para desenvolvimento de aplicativos (MICAz Datasheet 2006). Os motes MICA2, IRIS e TelosB, da MEMSIC, são menos utilizados, o que sugere que MICAz tem sido o mais difundido no âmbito de pesquisas. 


\section{DESAFIOS E DIFICULDADES NA IMPLEMENTAÇÃO DE PROTÓTIPOS REAIS}

Dentre os projetos apresentados, uma parte realizou instalação de protótipo em ambientes reais, enquanto outros se limitaram a testes em ambientes controlados (testbeds) ou com simulações computadorizadas. Dentre as implementações com ambientes reais, podem ser destacados diversos desafios e dificuldades.

Um dos desafios é a necessidade de balanço entre energia consumida e desempenho em redes de sensores sem fio, pois, apesar de medições em amostragens maiores trazerem dados mais precisos, podem trazer também um grande tráfego de dados, de acordo com (Rosi et al 2011). Tal trabalho teve problemas com a energia consumida pelos nós responsáveis pela transmissão dos dados para a estação base, e solucionou o problema com o uso de baterias em paralelo ao invés de em série. Dessa forma, concluiu que a maior influência no consumo de energia é o período de tempo em que o rádio é ligado, não a quantidade de dados transmitidos por pacote. O trabalho de (Intrieri et al 2018), por sua vez, teve problemas por conta de baterias, que duravam apenas semanas com medição horária de dados.

O problema de bateria também esteve presente em (Bednarczyk 2018), que utilizou energia solar para amenizá-lo, e (Giorgetti et al 2014), que desenvolveu um novo protocolo de rede para configuração autônoma de parâmetros de transmissão e aquisição de dados, além de um sistema para alteração manual dos parâmetros à distância. Já (Homma et al 2017) desenvolveu uma rede em que os nós mandam pacotes periódicos informando sua quantidade de energia e inclinação, além de realizarem medições mais constantes apenas em caso de grande variação da aceleração, o que permite que haja economia de energia.

Os locais em que os nós são colocados no ambiente de interesse é de grande relevância. A presença de solos instáveis torna essa tarefa mais complicada por conta da resistência a danos físicos dos nós. O trabalho de (Uchimura et al 2015) envolveu este problema, chegando a conclusão de que os locais dos sensores na área de monitoramento não podia ser decidido de forma definitiva por conta da dificuldade de se definir a localização exata das regiões instáveis do solo; de acordo com os autores, pode-se amenizar o problema com a instalação de nós sensores simples e de baixo custo em regiões com inclinações possivelmente instáveis. O artigo de (Ramesh \& Vasudevan 2012) descreve um protótipo que é instalado sob o solo para detecção. O projeto também sofreu com solos instáveis, pois houve a preocupação de que a futura retirada do protótipo de nó da terra pudesse trazer um colapso a um poço de água contido sob a região de instalação.

Em relação à escolha da melhor topologia de rede, sabe-se que ela está relacionada a diversos fatores da qualidade da rede. O projeto de (Supekar \& Takayama 2016) considerou para pesquisas da qualidade de sua rede aspectos relacionados a topologias como robustez da rede (relacionada ao número de canais de comunicação possuídos por nós), latência dos dados e balanceamento das cargas. Tais fatores são importantes, pois, dependendo da topologia, a confiabilidade dos dados pode ser afetada. Já em (Homma et al 2017), a rede auto-organizável altera sua topologia de acordo com o estado do sinal de comunicação entre os nós, permitindo que o sistema encontre os melhores caminhos para transmissão de dados.

O problema de danos físicos a nós da rede foi citado por diversos trabalhos, como o de (Rosi et al 2011), no qual os autores citam que o mal posicionamento dos nós na região pode fazer com que sejam enterrados ou cobertos pela vegetação, impedindo que consigam enviar dados. Uma questão diretamente relacionada é a escolha do invólucro dos nós: no projeto, os nós foram envoltos, por conta da umidade, com um pacote plástico, o qual foi furado na parte de baixo para permitir medições de dados do solo e permitir a retirada de água condensada que possivelmente pudesse estar na parte de dentro do invólucro. Além disso, para cada nó foi construída uma cobertura de madeira inserida no solo com barras de ferro para proteção contra chuvas, atividade selvagem, movimentos de inclinação do solo e quedas de pedras.

Outro fator é a escolha entre diferentes redes para transmissão de dados por conta de latência e sinal de transmissão. Por exemplo, (Huo et al 2011) aborda o projeto Landslides Remote Monitoring System, que visa o monitoramento de áreas remotas e utiliza redes GSM; é citado que a escolha do GSM traz problemas por conta de sinais fracos devido à localização dos nós em áreas afastadas. Além disso, o GSM trouxe cerca de 40s de atraso na transmissão dos dados, o que pode prejudicar a entrega de dados e trazer perda de pacotes.

Por fim, o desenvolvimento das redes deve visar a detecção de deslizamentos em ambientes reais. O trabalho de (Intrieri et al 2012) ressaltou a dificuldade apresentada para estabelecer limites de deslizamentos de forma precisa. Isto ocorreu pela simplicidade dos procedimentos aplicados após um ano de atividade da rede, pois neste período apenas em uma vez obteve-se nível de atenção, em que a ocorrência de um deslizamento pode estar próxima. Dentre estes procedimentos estão a média dos dados medidos para evitar picos de nós específicos e o uso de redundância, ou seja, dois ou mais nós devem exceder seus limiares para o sistema em nível de atenção. Além disso, houve avaliação por especialistas do nível do alarme. 


\section{CONCLUSÃO}

Este trabalho propôs uma revisão sistemática da área de redes de sensores sem fio para monitoramento e detecção de deslizamentos de terra, propondo discussões sobre tópicos diversos. Algumas das principais conclusões obtidas foram que os equipamentos mais utilizados de hardware são o mote MICAz, os acelerômetros e os sensores de umidade e temperatura. Além disso, a topologia em malha se mostrou a mais utilizada, e a transmissão de dados pela rede ocorre principalmente via rádio.

Além disso, foram abordados problemas e dificuldades na implementação em ambientes reais de redes de sensores sem fio. A discussão envolveu alguns dos tópicos mais presentes nos projetos estudados, como energia, transmissão de dados, topologia de rede, resistência a danos físicos pelos nós e o problema de se estabelecer limiares de ocorrência de deslizamentos. Espera-se que esta revisão auxilie o desenvolvimento de novos projetos e protótipos de redes de sensores voltados ao tema. Por fim, trabalhos futuros incluem o desenvolvimento de um protótipo de rede para detecção de deslizamentos a partir das informações obtidas com a revisão sistemática e com os estudos dos desafios e dificuldades de projetos existentes.

\section{AGRADECIMENTO}

Esta pesquisa é parte do INCT da Internet do Futuro para Cidades Inteligentes financiado pelo CNPq proc. 465446/2014-0, Coordenação de Aperfeiçoamento de Pessoal de Nível Superior - Brasil (CAPES) - Código de Financiamento 001, FAPESP proc. 14/50937-1, e FAPESP proc. 15/24485-9.

\section{REFERÊNCIAS}

Branco, A., Sant'Anna, F., Ierusalimschy, R., Rodriguez, N. and Rossetto, S., 2015. Terra: Flexibility and safety in wireless sensor networks. ACM Transactions on Sensor Networks (TOSN), 11(4), p.59.

Cemaden 2019. Cemaden - Centro Nacional de Monitoramento e Alertas de Desastres Naturais. Acessado em 24 de setembro de 2019, <https://www.cemaden.gov.br/>.

Homma, M., Nakano, T., van Khoa, V. and Takayama, S., 2017, November. Characteristic functions of wireless sensing node network for landslide disaster. In TENCON 2017-2017 IEEE Region 10 Conference (pp. 706-711). IEEE.

Huo, Z., Zhang, P., Tao, Z. and Gui, Y., 2011, September. Landslides Remote Monitoring System and its shortages appeared in applications. In 2011 International Conference on Electrical and Control Engineering (pp. 1603-1606). IEEE.

IBGE. Pesquisa de informações básicas municipais: 2013. (2014). Rio de Janeiro.

Intrieri, E., Gigli, G., Mugnai, F., Fanti, R. and Casagli, N., 2012. Design and implementation of a landslide early warning system. Engineering Geology, 147, pp.124-136.

MICAz Datasheet 2006, Crossbow technology inc. Acessado em 24 de setembro de 2019, <http://www.openautomation.net/uploadsproductos/micaz_datasheet.pdf>

Ramesh, M.V. and Vasudevan, N., 2012. The deployment of deep-earth sensor probes for landslide detection. Landslides, 9(4), pp.457-474.

Rosi, A., Berti, M., Bicocchi, N., Castelli, G., Corsini, A., Mamei, M. and Zambonelli, F., 2011. Landslide monitoring with sensor networks: experiences and lessons learnt from a real-world deployment. International Journal of Sensor Networks, 10(3), pp.111-122.

Uchimura, T., Towhata, I., Wang, L., Nishie, S., Yamaguchi, H., Seko, I. and Qiao, J., 2015. Precaution and early warning of surface failure of slopes using tilt sensors. Soils and Foundations, 55(5), pp.1086-1099 9. 